QCD Evolution Workshop 2014

International Journal of Modern Physics: Conference Series

Vol. 37 (2015) 1560057 (8 pages)

(C) The Author

DOI: $10.1142 / \mathrm{S} 2010194515600575$

\title{
High Energy Resummation: BFKL vs Sudakov
}

\author{
Feng Yuan \\ Nuclear Science Division, Lawrence Berkeley National Laboratory, \\ Berkeley, CA 94720, USA \\ fyuan@lbl.gov
}

Published 25 February 2015

\begin{abstract}
In this talk, we review recent theoretical developments on QCD resummation in high energy scattering processes in $p p$ and $p A$ collisions. In particular, we will show that the transverse momentum resummation (Sudakov) can be performed consistently in the small- $x$ factorization formalism (BFKL) at the leading double logarithmic approximation. We further discuss the physics beyond the above approximation, and show that the TMD resummation at next-to-leading logarithmic order can describe the dijet production experimental data from D0 collaboration. Finally, we comment on the TMD resummation effects in the so-called Mueller-Navelet jet production in hadronic collisions.
\end{abstract}

Keywords: QCD evolution; transverse momentum dependent parton distributions.

PACS numbers: 12.38.Bx, 12.39.St, 13.85.Qk

\section{Introduction}

An important application of the perturbative quantum chromodynamics (QCD) is the resummation. In high energy hadronic processes involving largely separated scales, resummation is not only necessary to make reliable predictions, but also crucial to extract the fundamental properties of the strong interaction theory. One of the examples is the resummation of the Sudakov-type double logarithms $[1,2]$. The double logarithms appear in, for example, the transverse momentum spectrum of a hard process, where each order of perturbative correction is accompanied by a large double logarithmic term of $\ln ^{2}\left(Q^{2} / k_{\perp}^{2}\right)$ with $Q$ the large momentum scale and $k_{\perp}$ the transverse momentum. This resummation is often referred as the transverse momentum dependent (TMD) resummation. Meanwhile, there is also the small- $x$ resummation which is equally important, in particular, in the energy regime of the large hadron collider (LHC). The small- $x$ resummation is governed by the wellknown BFKL evolution [3], which will be extended to the so-called BK-JIMWLK

This is an Open Access article published by World Scientific Publishing Company. It is distributed under the terms of the Creative Commons Attribution 3.0 (CC-BY) License. Further distribution of this work is permitted, provided the original work is properly cited. 
evolution $[4,5,6]$ to take into account the non-linear term in the evolution due to high gluon density in nucleons/nuclei at high energy.

One would naturally ask the following question: if there is a place that we have to resum the above two large logarithms and can we do that consistently? The answer is yes. In recent studies of Refs. [7, 8], it has been shown that we can perform the above two resummations (Sudakov and BFKL) consistently in physical processes in high energy scattering. In this talk, we will present a brief overview of these results. First, we show that the Sudakov double logarithms can be resummed consistently in the small- $x$ factorization formalism. That is to say, we can have both resummations in the hard processes in $p A$ collisions. Second, we present the calculations beyond the leading double logarithmic approximation, where the TMD resummation has to take a more complicated form. We will also show that the resummation formula can describe the experimental data from D0 collaboration. Finally, we discuss the case of dijet production with rapidities well separated in $p p$ collisions, and comment on the role of the TMD resummation for the so-called Mueller-Navelet dijet production.

\section{Sudakov Leading Double Logarithms Resummation in Small-x Formalism}

Dijet production in forward $p A$ collisions can be used to probe the un-integrated gluon distributions in nucleus [9]. To be more precise, the dijet configuration with transverse momenta $k_{1 \perp}$ and $k_{2} \perp$ that we are especially interested in is the almost back-to-back dijet configuration, in which we can define two interesting variables, i.e., the dijet transverse momentum imbalance $q_{\perp} \equiv k_{1 \perp}+k_{2}$ and the dijet relative transverse momentum $P_{\perp} \equiv \frac{1}{2}\left|k_{1 \perp}-k_{2}\right|$ with $P_{\perp}^{2} \gg q_{\perp}^{2}$. For example, we can write down a generic expression for the dijet production cross section depending on $q_{\perp}$ as

$$
\frac{d \sigma}{d y_{1} d y_{2} d P_{\perp}^{2} d^{2} q_{\perp}} \propto H\left(P_{\perp}^{2}\right) \int d^{2} x_{\perp} d^{2} y_{\perp} e^{i q_{\perp} \cdot\left(x_{\perp}-y_{\perp}\right)} \widetilde{W}_{x_{A}}\left(x_{\perp}, y_{\perp}\right)
$$

where $H\left(P_{\perp}\right)$ represents the hard factor only depending on the hard momentum scale $P_{\perp}$ and the rapidities of the two jets $\left(y_{1}\right.$ and $\left.y_{2}\right), \widetilde{W}_{x_{A}}$ represents the Wilson lines associated with the un-integrated gluon distributions of nucleus. In the correlation limit of dijet production, $P_{\perp}$ dependence is decoupled from $q_{\perp}$ dependence, and the differential cross section can be directly calculated from the unintegrated gluon distributions of the nucleus, which obey the small- $x$ evolution equations. Solving these evolution equations, we resum the large logarithms associated with $\ln (1 / x)=\ln \left(s / P_{\perp}^{2}\right)$. In addition to the small- $x$ logarithms, the appearance of Sudakov double logarithms, i.e., $\bar{\alpha}_{s} \ln ^{2}\left(P_{\perp}^{2} / q_{\perp}^{2}\right)$, requires another type of resummation, namely, the Sudakov resummation $[1,2]$. Therefore, it is important to know whether or not we can consistently compute and perform the Sudakov resummation in the saturation formalism, in the presence of small- $x$ resummation. 
In Refs. [7, 8], this question was answered, and it has been shown that the Sudakov double logarithmic terms and small- $x$ type logarithms can be resumed simultaneously and independently in the saturation formalism. This conclusion holds for many processes, such as Higgs boson production, heavy quark pair productions and back-to-back dijet production in $p A$ collisions as well as in DIS.

After taking into account the Sudakov double logarithms resummation, the differential cross section of Eq. (1) can be modified as

$$
\frac{d \sigma}{d y_{1} d y_{2} d P_{\perp}^{2} d^{2} q_{\perp}} \propto H\left(P_{\perp}^{2}\right) \int d^{2} x_{\perp} d^{2} y_{\perp} e^{i q_{\perp} \cdot R_{\perp}} e^{-\mathcal{S}_{s u d}\left(P_{\perp}, R_{\perp}\right)} \widetilde{W}_{x_{A}}\left(x_{\perp}, y_{\perp}\right)
$$

where the Sudakov factor can be written as

$$
\mathcal{S}_{\text {sud }}=\frac{\alpha_{s}}{\pi} \mathcal{C} \int_{c_{0}^{2} / R_{\perp}^{2}}^{P_{\perp}^{2}} \frac{d \mu^{2}}{\mu^{2}} \ln \frac{P_{\perp}^{2}}{\mu^{2}} .
$$

where $c_{0}=2 e^{-\gamma_{E}}$ is defined in the following calculations and $R_{\perp}=x_{\perp}-y_{\perp}$. The $\mathcal{C}$ coefficient is identified in the Sudakov double logarithmic factor, which depends on the color flow. The color factor $\mathcal{C}$ is process dependent, and has to be determined case by case for various dijet production processes.

We emphasize that the small- $x$ resummation of high order corrections in terms of $\ln \left(s / P_{\perp}^{2}\right) \sim \ln \left(1 / x_{A}\right)$ is carried out by solving the small- $x$ evolution equations for the associated un-integrated gluon distributions $\widetilde{W}_{x_{A}}\left(x_{\perp}, y_{\perp}\right)$, whereas the Sudakov double logarithms in terms of $\ln \left(P_{\perp}^{2} / q_{\perp}^{2}\right)$ is resummed by the Sudakov form factor $e^{-\mathcal{S}_{\text {sud }}}$ in the above equations. Therefore, the above equations resummed both small$x$ logarithms and Sudakov logarithms simultaneously.

The physics behind the above results can be understood as follows. The gluon radiation comes from three different regions, for a particular partonic channel in $p A$ collisions: (1) collinear gluon parallel to the incoming nucleon; (2) collinear gluon parallel to the incoming nucleus; (3) soft gluon radiation. For example, for onegluon radiation contribution to the Born diagram of the above hard processes in $p A$ collisions, we can parameterize the radiated gluon momentum as

$$
q=\alpha_{q} p_{1}+\beta_{q} p_{2}+q_{\perp},
$$

where $p_{1}$ and $p_{2}$ denote the four momenta of incoming partons in the partonic process. Since the phase space of the radiated gluon will be integrated out to obtain the final differential cross section, we need to consider the three different momentum regions discussed above. The momentum region of (1) corresponds to $\alpha_{q} \sim \mathcal{O}(1)$, whereas $\beta_{q} \ll 1$, which contributes to the collinear divergence associated with the parton distribution from the nucleon. For region (2), the dominant collinear gluon radiation parallel to the nucleus requires not only $\beta_{q}$ of order 1 , but also close to 1 , i.e., $1-\beta_{q} \ll 1$. Effectively, because of $q_{\perp} \sim q_{\perp} \ll M\left(P_{\perp}\right)$, this leads to $\alpha_{q} \rightarrow 0$. This corresponds to the rapidity divergence at one-loop order, which can be absorbed into the renormalization of the un-integrated gluon distribution of the nucleus in the small- $x$ limit. Region (3) concerns the Sudakov double logarithms 
where $\alpha_{q} \sim \beta_{q} \ll 1$. The gluon radiation in this kinematic region depends on the overall color flow in the hard partonic processes. That is why the Sudakov double logarithms have simple counting rule [8]. The kinematics of the three regions are well separated, and at the leading power of $q_{\perp} / M\left(P_{\perp}\right)$, they can be factorized into various factors.

Both Sudakov effect (represented by $\mathcal{S}_{\text {sud }}$ ) and small-x effect (represented by $S_{Y}^{W W}$ ) can generate the $p_{\perp}$-broadening in hard processes. From the result in Eq. (2), we can see that these two effects can be consistently included. As a consequence, we immediately find that the Sudakov effects will be dominant effects in the dilute region where $Q_{s} \ll Q$. On the other hand, in the dense region with $Q \sim Q_{s}$, the small- $x$ effects dominates. In the kinematics of di-hadron correlations in the forward direction of $d A u$ collisions at RHIC, the saturation scale is large enough, and thus we can safely neglect the Sudakov effects [10].

\section{Beyond Leading Logarithmic Approximation}

In the above discussions, we have shown that the TMD resummation is simplified in the leading double logarithmic approximation. Beyond that, the resummation takes a much more complicated form. Again, for dijet production, the resummation formula can be summarized as

$$
\frac{d^{4} \sigma}{d y_{1} d y_{2} d P_{\perp}^{2} d^{2} q_{\perp}}=\sum_{a b} \sigma_{0}\left[\int \frac{d^{2} \vec{b}_{\perp}}{(2 \pi)^{2}} e^{-i \vec{q}_{\perp} \cdot \vec{b}_{\perp}} W_{a b \rightarrow c d}\left(x_{1}, x_{2}, b_{\perp}\right)+Y_{a b \rightarrow c d}\right]
$$

where the first term $W$ contains all order resummation and the second term $Y$ comes from the fixed order corrections; $\sigma_{0}$ represents normalization of the differential cross section, $y_{1}$ and $y_{2}$ are rapidities of the two jets, $P_{\perp}$ is the leading jet transverse momentum, and $q_{\perp}$ the imbalance transverse momentum between the two jets as defined above. All order resummation for $W$ from each partonic channel $a b \rightarrow c d$ can be written as [11]

$$
\begin{aligned}
W_{a b \rightarrow c d}\left(x_{1}, x_{2}, b\right)= & x_{1} f_{a}\left(x_{1}, \mu=b_{0} / b_{\perp}\right) x_{2} f_{b}\left(x_{2}, \mu=b_{0} / b_{\perp}\right) e^{-S_{\text {Sud }}\left(Q^{2}, b_{\perp}\right)} \\
& \times \operatorname{Tr}\left[\mathbf{H}_{a b \rightarrow c d} \exp \left[-\int_{b_{0} / b_{\perp}}^{Q} \frac{d \mu}{\mu} \gamma^{s \dagger}\right] \mathbf{S}_{a b \rightarrow c d}\right. \\
& \left.\times \exp \left[-\int_{b_{0} / b_{\perp}}^{Q} \frac{d \mu}{\mu} \gamma^{s}\right]\right],
\end{aligned}
$$

where $Q^{2}=\hat{s}=x_{1} x_{2} S$, which represents the hard momentum scale, $b_{0}=2 e^{-\gamma_{E}}$, $f_{a, b}(x, \mu)$ are parton distributions for the incoming partons $a$ and $b, x_{1,2}=$ $P_{\perp}\left(e^{ \pm y_{1}}+e^{ \pm y_{2}}\right) / \sqrt{S}$ are momentum fractions of the incoming hadrons carried by the partons. In the above equation, the hard and soft factors $\mathbf{H}$ and $\mathbf{S}$ are expressed as matrices in the color space of partonic channel $a b \rightarrow c d$, and $\gamma^{s}$ are the associated anomalous dimensions for the soft factor (defined below). The Sudakov form 
factor $\mathcal{S}_{\text {Sud }}$ resums the leading double logarithms and the universal sub-leading logarithms,

$$
S_{\mathrm{Sud}}\left(Q^{2}, b_{\perp}\right)=\int_{b_{0}^{2} / b_{\perp}^{2}}^{Q^{2}} \frac{d \mu^{2}}{\mu^{2}}\left[A \ln \left(\frac{Q^{2}}{\mu^{2}}\right)+B+D_{1} \ln \frac{Q^{2}}{P_{\perp}^{2} R_{1}^{2}}+D_{2} \ln \frac{Q^{2}}{P_{\perp}^{2} R_{2}^{2}}\right]
$$

where $R_{1,2}$ represent the cone sizes for the two jets. Here the parameters $A, B$, $D_{1}, D_{2}$ can be expanded perturbatively in $\alpha_{s}$. At one-loop order, $A=C_{A} \frac{\alpha_{s}}{\pi}$, $B=-2 C_{A} \beta_{0} \frac{\alpha_{s}}{\pi}$ for gluon-gluon initial state, $A=C_{F} \frac{\alpha_{s}}{\pi}, B=\frac{-3 C_{F}}{2} \frac{\alpha_{s}}{\pi}$ for quarkquark initial state, and $A=\frac{\left(C_{F}+C_{A}\right)}{2} \frac{\alpha_{s}}{\pi}, B=\left(\frac{-3 C_{F}}{4}-C_{A} \beta_{0}\right) \frac{\alpha_{s}}{\pi}$ for gluon-quark initial state. These coefficients $A$, at the one-loop order, follow the power counting analysis $[12,8]$.

At the next-to-leading logarithmic level, the jet cone size enters as well [11]. That is the reason we have two additional factors in Eq. (7): $D=C_{A} \frac{\alpha_{s}}{2 \pi}$ for gluon jet and $D=C_{F} \frac{\alpha_{s}}{2 \pi}$ for quark jet. The cone size $R$ is introduced to regulate the collinear gluon radiation associated with the final state jets. Only the soft gluon radiation outside the jet cone contributes to the imbalance $q_{\perp}$ between the two jets, and yields the logarithmic contribution in the form of $\ln \left(\frac{Q^{2}}{P_{\perp}^{2} R^{2}}\right)$. This conclusion is independent of the jet algorithm [13]. In Fig. 1, the resummation results are compared to the experimental data from D0 Collaboration at the Tevatron [14]. When Fourier transforming the $b_{\perp}$-expression to obtain the transverse momentum distribution, we follow the $b_{*}$ prescription of CSS resummation [2], i.e., replacing $b_{\perp}$ by $b_{*}=b / \sqrt{1+b^{2} / b_{\max }^{2}}$ in the calculation. By doing so, we will also introduce

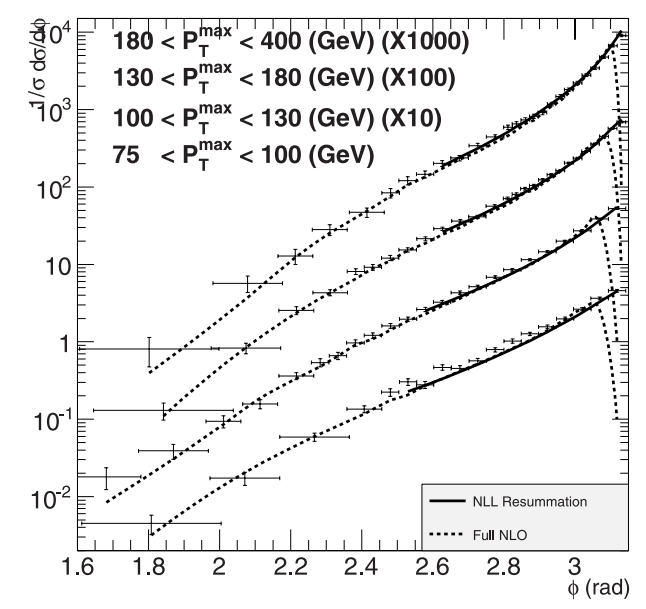

Fig. 1. Resummation results on dijet azimuthal correlations at the Tevatron, compared to the experimental data from D0 Collaboration, where both jets are produced in central-rapidity region with $|y|<0.5$. Away from $\phi=\pi$, the resummation results (solid curves) are matched to a full NLO calculation (dashed curves). 
the non-perturbative form factors for the quarks and gluons from the initial states. In our calculations, we have used $b_{\max }=0.5 \mathrm{GeV}^{-1}$, and the non-perturbative form factors follow the parameterizations in Refs. [15]. However, it was found that because the jet energy is so large that our final results are not sensitive to the non-perturbative form factors at all.

From Fig. 1, we see that the resummation results agree well with the experimental data, from $\phi$ near to $\pi$ down to much smaller values. For smaller value of $\phi$ (away from the back-to-back configuration), the resummation calculations match to the fixed order results at NLO [16], which has also been separately shown in Fig. 1. We note that a full NLO calculation cannot describe experimental data for $\phi \sim \pi[14]$, where the fixed order calculation becomes divergent. Our resummation calculation, after being matched with the NLO result. clearly improves the theory prediction and can describe the experimental data in a wider kinematic region. This demonstrates the importance of all order resummation in perturbative calculations for these type of hard QCD processes.

\section{TMD Resummation in Mueller-Navelet Dijet Production}

We also notice that recently, the CMS collaboration at the LHC has measured the dijet azimuthal correlation with large rapidity separation between the jets (MuellerNavelet dijet), which has been interpreted as the BFKL resummation effects [17]. In the following, we briefly discuss the TMD resummation effects in the MuellerNavelet dijet production. As a first step, we examine the calculations of one-gluon radiation contribution to Mueller-Navelet dijet Production in the collinear framework, and argue the associated resummations at higher orders, including both Sudakov type and BFKL type when the rapidity between the two jets become large. We take the quark-quark scattering channel as an example. At the leading order,

$$
\frac{d \sigma(q q \rightarrow q q)}{d k_{\perp}^{2}} \propto \frac{\alpha_{s}^{2}}{16 \pi^{2} s^{2}} \frac{s^{2}+u^{2}}{t^{2}}
$$

where $s, t, u$ as defined usual, and $k_{\perp}^{2}=t u / s$ is the transverse momentum of final state quark jet. In the leading order contribution, the balance momentum between the two jets equals to zero, $\vec{q}_{\perp}=\vec{k}_{1 \perp}+\vec{k}_{2 \perp}=0$.

At one-loop order, the most important contributions come from collinear and soft gluon radiations. The collinear gluons can be easily calculated, and they are proportional to collinear quark splitting. The soft gluon radiation can be summarized as $[8,11]$,

$$
\begin{aligned}
\frac{\alpha_{s}}{2 \pi^{2}} & \frac{1}{k_{g \perp}^{2}}\left\{2 C_{F} \ln \frac{Q^{2}}{k_{g \perp}^{2}}+C_{F}\left[\ln \frac{1}{R_{1}^{2}}+\ln \frac{1}{R_{2}^{2}}\right]\right. \\
& \left.+2 C_{F} \ln \frac{u}{t}+\frac{1}{N_{C}}\left(2 \ln \frac{s}{-t}+2 \ln \frac{s}{-u}\right)\right\},
\end{aligned}
$$


where $Q^{2}=s, k_{g \perp}$ is the radiated gluon transverse momentum, and we have used cone sizes $R_{1}$ and $R_{2}$ to regulate the collinear contribution associated with final state jets. We have only kept the leading and subleading contributions in the limit of $k_{g \perp} \ll k_{\perp}$. At one-loop order, $k_{g \perp}=q_{\perp}$ and contributes to a nonzero imbalance between the two jets.

In the discussions of Mueller-Navelet jets productions, the two jets in the final state are well separated in the rapidity. In other words, the partonic $s$ is much larger than $-t$. Therefore, we will apply the following approximation to evaluate the leading contribution for Mueller-Navelet dijet,

$$
s \sim-u \gg-t .
$$

Under the above limit, we find that Eq. (9) can be simplified as,

$$
\frac{\alpha_{s}}{2 \pi^{2}} \frac{1}{k_{g \perp}^{2}}\left\{2 C_{F} \ln \frac{Q^{2}}{k_{g \perp}^{2}}+C_{F}\left[\ln \frac{1}{R_{1}^{2}}+\ln \frac{1}{R_{2}^{2}}\right]+\left(C_{A}+\frac{1}{N_{c}}\right) \ln \frac{Q^{2}}{k_{\perp}^{2}}\right\},
$$

where we have used the fact that $s^{2} / t u=s / k_{\perp}^{2}$ and $u / t \approx s / k_{\perp}^{2}$. Now, everything is factorized out, if we rewrite the above result,

$$
\frac{\alpha_{s}}{2 \pi^{2}} \frac{1}{k_{g \perp}^{2}}\left\{2 C_{F} \ln \frac{k_{\perp}^{2}}{k_{g \perp}^{2}}+C_{F}\left[\ln \frac{1}{R_{1}^{2}}+\ln \frac{1}{R_{2}^{2}}\right]+2 C_{A} \ln \frac{Q^{2}}{k_{\perp}^{2}}\right\},
$$

where the first term corresponds to the Sudakov double logs, the second term for the jet functions, and the third term to the BFKL small-x reusmmation term. We can also identify the third term depending on the rapidity separation between the two jets,

$$
\ln \frac{Q^{2}}{k_{\perp}^{2}} \sim \Delta y
$$

The above equation tells us that the Sudakov physics and BFKL physics are well separated. And at this order, there is nothing else. That supports that we do have a factorization to separately resum the large logs [18].

\section{Conclusion}

In this talk, we have reviewed recent theoretical and phenomenological developments on QCD resummation in hard processes in hadronic collisions, in particular, for dijet productions. It was shown that the two famous resummations in high energy scattering: Sudakov and BFKL can be encoded in the calculations consistently. This has opened a new window to study strong interaction physics in great details. We anticipate more theoretical developments along this direction.

This material is based upon work supported by the U.S. Department of Energy, Office of Science, Office of Nuclear Physics, under contracts No. DE-AC02$05 \mathrm{CH} 11231$. 


\section{References}

1. V. V. Sudakov, Sov. Phys. JETP 3, 65 (1956) [Zh. Eksp. Teor. Fiz. 30, 87 (1956)]; Y. L. Dokshitzer, D. Diakonov and S. I. Troian, Phys. Rept. 58, 269 (1980); G. Parisi and R. Petronzio, Nucl. Phys. B 154, 427 (1979).

2. J. C. Collins, D. E. Soper and G. F. Sterman, Nucl. Phys. B 250, 199 (1985).

3. I. I. Balitsky and L. N. Lipatov, Sov. J. Nucl. Phys. 28, 822 (1978) [Yad. Fiz. 28, 1597 (1978)]; E. A. Kuraev, L. N. Lipatov and V. S. Fadin, Sov. Phys. JETP 45, 199 (1977) [Zh. Eksp. Teor. Fiz. 72, 377 (1977)]; A. H. Mueller, Nucl. Phys. B 415, 373 (1994).

4. I. Balitsky, Nucl. Phys. B463, 99-160 (1996); Y. V. Kovchegov, Phys. Rev. D60, 034008 (1999).

5. J. Jalilian-Marian, A. Kovner, A. Leonidov and H. Weigert, Nucl. Phys. B 504, 415 (1997); J. Jalilian-Marian, A. Kovner, A. Leonidov and H. Weigert, Phys. Rev. D 59, 014014 (1998).

6. E. Iancu, A. Leonidov and L. D. McLerran, Nucl. Phys. A 692, 583 (2001); Nucl. Phys. A 703, 489 (2002).

7. A. H. Mueller, B. W. Xiao and F. Yuan, Phys. Rev. Lett. 110, 082301 (2013).

8. A. H. Mueller, B. W. Xiao and F. Yuan, Phys. Rev. D 88, no. 11, 114010 (2013).

9. F. Dominguez, B. W. Xiao and F. Yuan, Phys. Rev. Lett. 106, 022301 (2011); F. Dominguez, C. Marquet, B. W. Xiao and F. Yuan, Phys. Rev. D 83, 105005 (2011).

10. A. Stasto, B. -W. Xiao and F. Yuan, Phys. Lett. B 716, 430 (2012).

11. P. Sun, C.-P. Yuan and F. Yuan, arXiv:1405.1105 [hep-ph] (2014).

12. A. Banfi, M. Dasgupta and Y. Delenda, Phys. Lett. B 665, 86 (2008).

13. A. Mukherjee and W. Vogelsang, Phys. Rev. D 86, 094009 (2012).

14. V. M. Abazov et al. [D0 Collaboration], Phys. Rev. Lett. 94, 221801 (2005).

15. F. Landry, R. Brock, P. M. Nadolsky and C. P. Yuan, Phys. Rev. D 67, 073016 (2003); Phys. Rev. D 63, 013004 (2001); P. Sun, C. -P. Yuan and F. Yuan, Phys. Rev. D 88, 054008 (2013).

16. Z. Nagy, Phys. Rev. Lett. 88, 122003 (2002); Phys. Rev. D 68, 094002 (2003).

17. B. Duclou, L. Szymanowski and S. Wallon, Phys. Rev. Lett. 112, 082003 (2014).

18. B. Xiao, et al., to appear. 